\title{
Analysis of the conditions for the existence of stable microcracks in an elastic stress field from a rotational-shear mesodefect
}

\author{
S. V. Kirikov ${ }^{\dagger, 1,2}$, V.N. Perevezentsev ${ }^{1}$ \\ †ksv.kirikov@yandex.ru \\ ${ }^{1}$ Mechanical Engineering Research Institute of RAS, Nizhny Novgorod, 603024, Russia \\ ${ }^{2}$ National Research University "Higher School of Economics", Nizhny Novgorod, 603155, Russia
}

\begin{abstract}
During plastic flow, simultaneously with the formation of junction disclinations, which are linear mesodefects, planar shear mesodefects appear at the grain boundaries and facets of the boundaries, the stress fields from which can significantly affect the orientation and characteristics of a microcrack formed at the junction. The configurational force method is used to analyze the conditions for the existence of stable cracks in the elastic field of a combined mesodefect, which is a superposition of a wedge disclinations dipole and a planar mesodefect. In the configuration space of the parameters of the system under consideration (the strength of the disclinations dipole and the planar mesodefect, the length of the mesodefect, and the angle that specifies the orientation of the crack), the ranges of parameter values are determined at which such cracks can appear. The dependences of the critical strength of the disclination dipole and the length of the nuclear crack arising in the vicinity of the negative disclination of the dipole on the length of the mesodefect are calculated for different values of the strength of the planar mesodefect. It was assumed that the opening of the crack occurs in the direction coinciding with the orientation at which the length of the nuclear crack at fixed values of the strength of the disclinations dipole, the strength of the planar mesodefect and the length of the mesodefect is minimal, and, therefore, the energy for its creation is minimal. It is generally concluded that shear-type mesodefects can significantly facilitate the initiation of microcracks in the vicinity of junction disclinations. In the range of parameter values that allow the existence of stable cracks, the length of the nuclear crack decreases with increasing strength of the planar mesodefect. It is shown that the critical length of the crack is tenths of the length of the disclination dipole.
\end{abstract}

Keywords: dislocation, microcrack, disclination, plastic deformation.

УДК: 539.2

\section{Анализ условий существования стабильных микротрещин в упругом поле напряжений от ротационно-сдвигового мезодефекта}

\author{
Кириков С. В. ${ }^{\dagger, 1,2}$, Перевезенцев В.Н. ${ }^{1}$ \\ ${ }^{1}$ Институт проблем машиностроения РАН, Нижний Новгород, 603024, Россия \\ ${ }^{2}$ Национальный исследовательский университет «Высшая школа экономики», Нижний Новгород, 603155, Россия
}

В ходе пластической деформации одновременно с процессом образования стыковых дисклинаций, представляющих собой линейные мезодефекты, на границах зёрен и фасетках границ возникают и планарные мезодефекты сдвигового типа, поля напряжения от которых могут существенно повлиять на ориентацию и характеристики формирующейся в стыке микротрещины. С использованием метода конфигурационной силы проведён анализ условий существования стабильных трещин в упругом поле комбинированного мезодефекта, представляющего собой суперпозицию диполя клиновых дисклинаций и планарного мезодефекта. В конфигурационном пространстве параметров рассматриваемой системы (мощности диполя дисклинаций и планарного мезодефекта, длина мезодефекта и угол, задающий ориентацию трещины) определены области значений параметров, при которых возможно появление таких трещин. Рассчитаны зависимости критической мощности дисклинационного диполя и длины зародышевой трещины в окрестности отрицательной дисклинации диполя, от длины мезодефекта при различных значениях мощности планарного мезодефекта. Предполагалось, что раскрытие трещины происходит 
в направлении, совпадающем с такой ориентацией, при которой длина зародышевой трещины при фиксированных значениях мощности диполя дисклинаций, мощности планарного мезодефекта и длины мезодефекта минимальна, и следовательно, минимальны энергетические затраты на ее создание. Сделан общий вывод о том, что мезодефекты сдвигового типа могут существенно облегчать зарождение микротрещин в окрестности стыковых дисклинаций. В области значений параметров, допускающих возможность существования стабильных трещин, с ростом мощности планарного мезодефекта длина зародышевой трещины уменьшается. Показано, что критическая длина трещины составляет десятые доли от длины дисклинационного диполя.

Ключевые слова: дислокация, микротрещина, дисклинация, пластическая деформация.

\section{1. Введение}

Известно, что при пластической деформации поликристаллических твердых тел, вследствие неоднородности пластического течения по ансамблю зерен и неоднородности внутризеренной деформации, на границах и в стыках зерен формируются системы мезодефектов. В стыках зерен и в вершинах деформационных фасеток, возникающих на границах зёрен при их взаимодействии с потоками решёточных дислокаций, локализованными в полосах скольжения, появляются мезодефекты ротационного типа - стыковые дисклинации $[1,2]$. Мощность стыковых дисклинаций и создаваемые ими поля внутренних напряжений увеличиваются по мере пластического деформирования. При достаточно больших значениях истинной деформациях, вблизи дисклинаций формируются специфические аккомодационные структуры в виде оборванных стенок дислокаций, распространяющихся от стыков зёрен и вершин деформационных фасеток в тело зерен [1]. Этот процесс лежит в основе явления фрагментации материалов, то есть разбиения зерен на взаимно разориентированные области (фрагменты). По мере исчерпания процессов пластической аккомодации единственным каналом релаксации упругих напряжений от дисклинаций является зарождение микротрещин. В связи с этим становится очевидным, что важным этапом построения адекватной физической теории разрушения поликристаллических твёрдых тел, испытывающих большие пластические деформации, является разработка моделей зарождения и роста микротрещин вблизи ротационных мезодефектов. Так, условия появления микротрещин в упругом поле одиночной клиновой дисклинации рассматривались в работах $[3,4]$. Позднее аналогичные исследования были проведены для случая диполя клиновых дисклинаций и дисклинации, поле напряжений которой экранировано распределенным ансамблем дислокаций [5]. В развитии этих представлений в работах $[6,7]$ были проанализированы условия зарождения трещины Зинера-Гриффитса в суммарном поле внешних напряжений и внутренних напряжений от дисклинационного диполя.

Важно, однако, отметить, что в ходе пластической деформации одновременно с процессом образования стыковых дисклинаций, представляющих собой линейные мезодефекты, на границах зёрен и фасетках границ возникают и планарные мезодефекты сдвигового типа [8], поля напряжения от которых могут существенно повлиять на ориентацию и характеристики формирующейся в стыке микротрещины. Поэтому представляет интерес проанализировать условия появления микротрещин в упругом поле такого комбинированного мезодефекта. В настоящей работе это рассмотрение проведено для относительно простого случая мезодефекта, представляющего собой суперпозицию диполя клиновых дисклинаций и планарного мезодефекта.

\section{2. Описание модели}

Рассмотрим двухосный диполь клиновых дисклинаций мощности $w_{d p}$ с плечом $2 a$ (модуль мощности дисклинаций $\left.w_{d}=w_{d p}\right)$, содержащий планарный мезодефект, представляющий собой равномерно и континуально распределённые вдоль плеча диполя виртуальные скользящие краевые дислокации с плотностью вектора Бюргерса $w_{p l}$ (Рис. 1).

Такой изолированный мезодефект может возникать, например, на деформационной фасетке, которая образуется при прохождении полосы скольжения (или полосы сдвига) через границу наклона. В этом случае характеристики мезодефектов: $w_{p l}, w_{d p}, 2 a$ зависят от величины пластической деформации в полосе скольжения и ориентации полосы по отношению к границе зерна. Анализ взаимосвязи этих параметров представляет собой отдельную задачу. В данной работе мы абстрагируемся от истории возникновения рассматриваемого мезодефекта, и будем считать, его параметры независимыми друг от друга варьируемыми величинами.

Последовательный анализ условий, необходимых для образования микротрещины вблизи мезодефекта,

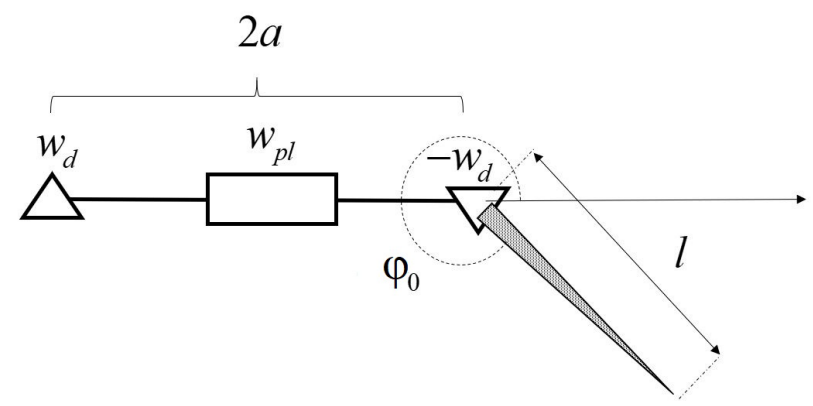

Рис. 1. Схематическое изображение комбинированного мезодефекта, состоящего из двухосного диполя клиновых дисклинаций (треугольники) и планарного мезодефекта (прямоугольник), с трещиной, зародившейся вблизи отрицательной дисклинации диполя.

Fig. 1. Schematic representation of a combined mesodefect consisting of a biaxial dipole of wedge disclinations (triangles) and a planar mesodefect (rectangle), with a crack that originated near the negative dipole disclination. 
включает в себя исследование стадии возникновения зародышевой трещины и условий ее раскрытия. В данной работе мы рассматриваем лишь условия, необходимые для существования в упругом поле рассматриваемого мезодефекта стабильной трещины (под стабильной трещиной далее понимается равновесная устойчивая трещина). Для этого удобно воспользоваться методом конфигурационной силы [9].

Рассмотрим трещину, расположенную вблизи отрицательной дисклинации диполя, создающей вблизи себя благоприятные для появления трещины высокие растягивающие напряжения (Рис. 1). В отсутствие планарного мезодефекта её ориентация совпадает с ориентацией дисклинационного диполя [5]. В другом случае $\left(w_{p l} \neq 0\right)$ трещина может располагаться под углом к диполю.

Для плоской деформации изотропного материала выражение для конфигурационной силы $F$, определяемой как величина упругой энергии, выделяющейся при продвижении трещины на единичный отрезок, имеет вид:

$$
F\left(l, \varphi=\varphi_{0}\right)=\frac{l}{8 D}\left(\bar{\sigma}_{\varphi \varphi}{ }^{2}+\bar{\sigma}_{r \varphi}{ }^{2}\right)
$$

где: $D=G /[2 \pi(1-v)], G-$ модуль сдвига, $v-$ коэффициент Пуассона, $l-$ длина трещины, $\varphi_{0}-$ полярный угол, задающий ориентацию рассматриваемой трещины (Рис. 1$), \bar{\sigma}_{\varphi \varphi}, \bar{\sigma}_{r \varphi}-$ средневзвешенные суммарные напряжения от диполя дисклинаций и планарного мезодефекта в окрестности трещины:

$$
\begin{aligned}
& \bar{\sigma}_{\varphi \varphi}=\frac{2}{\pi l} \int_{0}^{l} \sigma_{\varphi \varphi}\left(r, \varphi=\varphi_{0}\right) \sqrt{\frac{r}{l-r}} d r, \\
& \bar{\sigma}_{r \varphi}=\frac{2}{\pi l} \int_{0}^{l} \sigma_{r \varphi}\left(r, \varphi=\varphi_{0}\right) \sqrt{\frac{r}{l-r}} d r,
\end{aligned}
$$

где $\sigma_{\varphi \varphi}, \sigma_{r \varphi}-$ компоненты напряжения в полярной системе координат (полюс, которой совпадает с положением отрицательной дисклинации диполя), связанные с компонентами напряжений в декартовой системе соотношением: $\sigma_{P}=J^{-1} \sigma_{D} J$, где $\sigma_{P}-$ матрица тензора напряжений в полярной системе и $\sigma_{D}$ - матрица тензора напряжений в декартовой системе координат, $J$ - матрица Якоби, определяемая как:

$$
J=\left(\begin{array}{ll}
\vec{e}_{r}(1) & \vec{e}_{\varphi}(1) \\
\vec{e}_{r}(2) & \vec{e}_{\varphi}(2)
\end{array}\right)=\left(\begin{array}{cc}
\cos \varphi & -\sin \varphi \\
\sin \varphi & \cos \varphi
\end{array}\right),
$$

где

$$
\left\{\begin{array}{l}
\vec{e}_{r}=\frac{1}{\sqrt{\left|\frac{\partial \vec{R}}{\partial r}\right|}} \frac{\partial \vec{R}}{\partial r} \\
\vec{e}_{\varphi}=\frac{1}{\sqrt{\left|\frac{\partial \vec{R}}{\partial \varphi}\right|}} \frac{\partial \vec{R}}{\partial \varphi}
\end{array} .\right.
$$

Компоненты тензора упругих полей напряжений в правосторонней декартовой системе координат, начало которой совпадает с полюсом полярной системы, а ось $x$ направлена вдоль полярной оси, имеют вид: для планарного мезодефекта [10]

$$
\begin{aligned}
\frac{\sigma_{x x}}{D}= & \omega_{p l}\left(2\left(\arctan \left[\frac{x}{y}\right]-\arctan \left[\frac{x+2 a}{y}\right]\right)+\right. \\
& \left.+\frac{y(x+2 a)}{(x+2 a)^{2}+y^{2}}-\frac{y x}{x^{2}+y^{2}}\right), \\
\frac{\sigma_{y y}}{D}= & \omega_{p l}\left(\frac{x y}{x^{2}+y^{2}}-\frac{(x+2 a) y}{(x+2 a)^{2}+y^{2}}\right), \\
\frac{\sigma_{x y}}{D}= & \omega_{p l}\left(\frac{y^{2}}{(x+2 a)^{2}+y^{2}}-\frac{y^{2}}{x^{2}+y^{2}}+\right. \\
& \left.+\frac{1}{2} \ln \left[\frac{(x+2 a)^{2}+y^{2}}{x^{2}+y^{2}}\right]\right),
\end{aligned}
$$

для дисклинационного диполя [11]

$$
\begin{aligned}
\frac{\sigma_{x x}}{D} & =\omega_{d p}\left(\frac{y^{2}}{(x+2 a)^{2}+y^{2}}-\frac{y^{2}}{x^{2}+y^{2}}+\right. \\
& \left.+\frac{1}{2} \ln \left[\frac{(x+2 a)^{2}+y^{2}}{x^{2}+y^{2}}\right]\right), \\
\frac{\sigma_{y y}}{D} & =\omega_{d p}\left(\frac{y^{2}}{x^{2}+y^{2}}-\frac{y^{2}}{(x+2 a)^{2}+y^{2}}+\right. \\
& \left.+\frac{1}{2} \ln \left[\frac{(x+2 a)^{2}+y^{2}}{x^{2}+y^{2}}\right]\right), \\
\frac{\sigma_{x y}}{D} & =\omega_{d p}\left(\frac{x y}{x^{2}+y^{2}}-\frac{(x+2 a) y}{(x+2 a)^{2}+y^{2}}\right) .
\end{aligned}
$$

\section{3. Результаты расчетов и обсуждение}

Численные расчеты проводились при следующих значениях параметров: $G=45000 \mathrm{MПа,} v=0.3, \gamma=G b / 8$, $b=3 \cdot 10^{-4}$ мкм, $2 a=0.1-1$ мкм. Отметим, что выбранный диапазон значений длины дисклинационного диполя соответствует наблюдающемуся на эксперименте диапазону размеров деформационных фасеток [12].

Зависимость конфигурационной силы при фиксированных значениях параметров мезодефекта: $w_{d p}=w_{p l}=0.05$, $2 a=0.4$ мкм, от длины трещины $l$, рассчитанная при разных значениях угла $\varphi_{0}$, задающего ориентацию трещины, приведена на Рис. 2. Как видно из рисунка, в той области значений параметров системы, где конфигурационная сила $F(l) \geq 2 \gamma$ ( $\gamma-$ удельная энергия свободной поверхности), трещина размером $l \geq l_{0}$, самопроизвольно раскрывается. В дальнейшем находящуюся в положении неустойчивого равновесия трещину с длиной $l_{0}$ будем называть зародышевой. Величина $l_{0}$ существенно зависит от ориентации трещины. В дальнейшем будем полагать, что раскрытие трещины происходит в направлении, совпадающем с такой ориентацией $\varphi_{0}$, при которой длина зародышевой трещины $l_{0}\left(\varphi_{0}\right)$ минимальна и, следовательно, минимальны энергетические затраты на ее образование. При дальнейших расчетах этот критерий использовался для нахождения ориентации 
трещины при каждом конкретном наборе остальных параметров системы.

Величины $l_{0}$ и $l_{\text {eq }}$ зависят от характеристик дисклинационного диполя и планарного мезодефекта. Необходимое условие для появления вблизи рассматриваемого мезодефекта стабильной трещины можно записать в виде:

$$
\left.\max F(l, \varphi)\right|_{w_{d p}, w_{p l}, 2 a} \geq 2 \gamma
$$

Исходя из этого критерия, в конфигурационном пространстве $\left(w_{p l}, w_{d p}, 2 a\right)$ можно найти области значений параметров мезодефекта, при которых такая трещина может существовать. Граничная поверхность трехмерной области возможного существования трещины соответствует критическим параметрам мезодефекта: $\left(w_{p l}^{c r}, w_{d p}^{c r}, 2 a^{c r}\right)$ при которых выполняется соотношение:

$$
\left.\max F(l, \varphi)\right|_{w_{d p}^{c r}, w_{p l}^{c r}, 2 a^{c r}}=2 \gamma .
$$

На Рис. 3 а показаны зависимости критического значения $w_{d p}^{c r}$ от длины дисклинационного диполя $2 a$, полученные при различных значениях мощности планарного мезодефекта: $w_{p l}=0, w_{p l}=0.01, w_{p l}=0.02$. Области параметров, при которых возможно существование стабильных трещин, для каждого из приведенных значений $w_{p l}$ лежат выше соответствующих кривых $w_{d p}^{c r}(2 a)$. Видно, что величина критического значения $w_{d p}^{c r}$ уменьшается с ростом длины диполя дисклинаций и с увеличением мощности планарного мезодефекта. Влияние планарного мезодефекта на величину критического значения $w_{d p}^{c r}$, тем заметнее, чем больше длина диполя. Зависимости критической длины трещины от длины дисклинационного диполя при таких же значениях мощности планарного мезодефекта $w_{p l}$ приведены на Рис. 3 b.

Из приведенных данных следует, что при $w_{p l}=0$ относительная критическая длина трещины не меняется с увеличением плеча диполя и примерно равна 0.4. Увеличение мощности планарного мезодефекта приводит к появлению зависимости нормированной критической

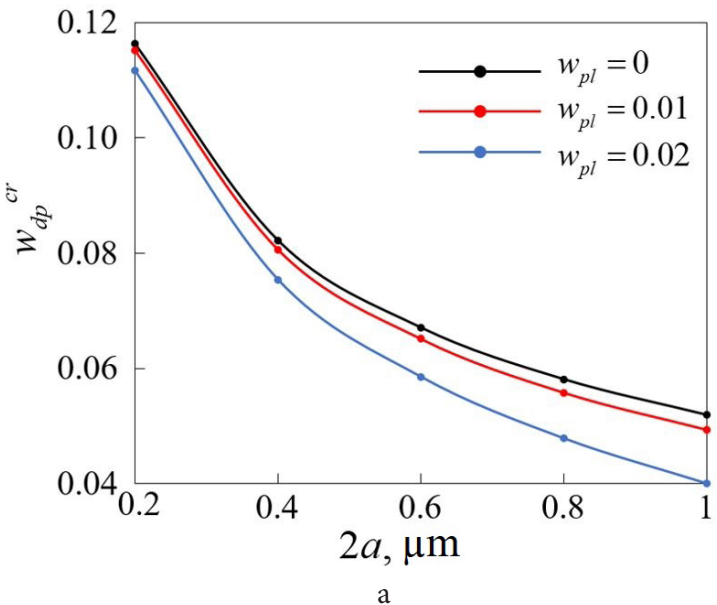



Рис. 2. Зависимости конфигурационной силы $F$ от длины формирующейся на мезодефекте трещины $l$, рассчитанные при разных значениях $\varphi_{0}$. Параметры мезодефекта: $w_{d p}=w_{p l}=0.05,2 a=0.4$ мКм.

Fig. 2. Dependences of the configuration force $F$ on the length of the crack $l$ formed on the mesodefect, calculated for different values of $\varphi_{0}$. Mesodefect parameters: $w_{d p}=w_{p l}=0.05,2 a=0.4 \mu \mathrm{m}$.

длины трещины $l^{c r} / 2 a$ от длины дисклинационного диполя. При этом $l^{c r} / 2 a$ убывает как при увеличении мощности планарного мезодефекта $w_{p l}$ так и при увеличении длины мезодефекта $2 a$. Как показывают расчеты, эта величина лежит в диапазоне значений 0.25 -0.4. Заметим, что при $w_{p l}=0$ ориентация трещины $\varphi_{0}$ не зависит от длины дисклинационного диполя и совпадает с ориентацией самого диполя. В тоже время при $w_{p l}>0$ эта зависимость появляется.

В области значений параметров системы, где выполняется неравенство $F(l) \geq 2 \gamma$, трещина длины $l>l$, самопроизвольно раскрывается и в отсутствии внешних напряжений достигает некоторой стабильной длины $l_{e q}$. На Рис. 4 приведены зависимости длины зародышевой трещины $l_{0}$ (Рис. $4 \mathrm{a}$ ) и длины стабильной трещины $l_{e q}$ (Рис. $4 \mathrm{~b}$ ) от мощности дисклинационного диполя длиной $2 a=0.6$ мкм при разных значениях мощности планарного мезодефекта $w_{p l}$.



Puc. 3. (Color online) Зависимости критической мощности диполя $w_{d p}^{c r}$ от длины диполя $2 a$ при разных значениях $w_{p l}=0, w_{p l}=0.01$, $w_{p l}=0.02$ (a) и нормированной критической длины трещины $l^{c r} / 2 a$ от длины диполя $2 a$ при разных значениях $w_{p l}^{p l}=0, w_{p l}^{p l}=0.01$, $w_{p l}=0.02(\mathrm{~b})$.

Fig. 3. (Color online) Dependences of critical strength of the dipople $w_{d p}^{c r}$ on its length $2 a$ at different values $w_{p l}=0, w_{p l}=0.01, w_{p l}=0.02$ (a) and normalized critical crack length $l^{c r} / 2 a$ on the length of the dipole $2 a$ at different values $w_{p l}=0, w_{p l}=0.01, w_{p l}=0.02$ (b). 

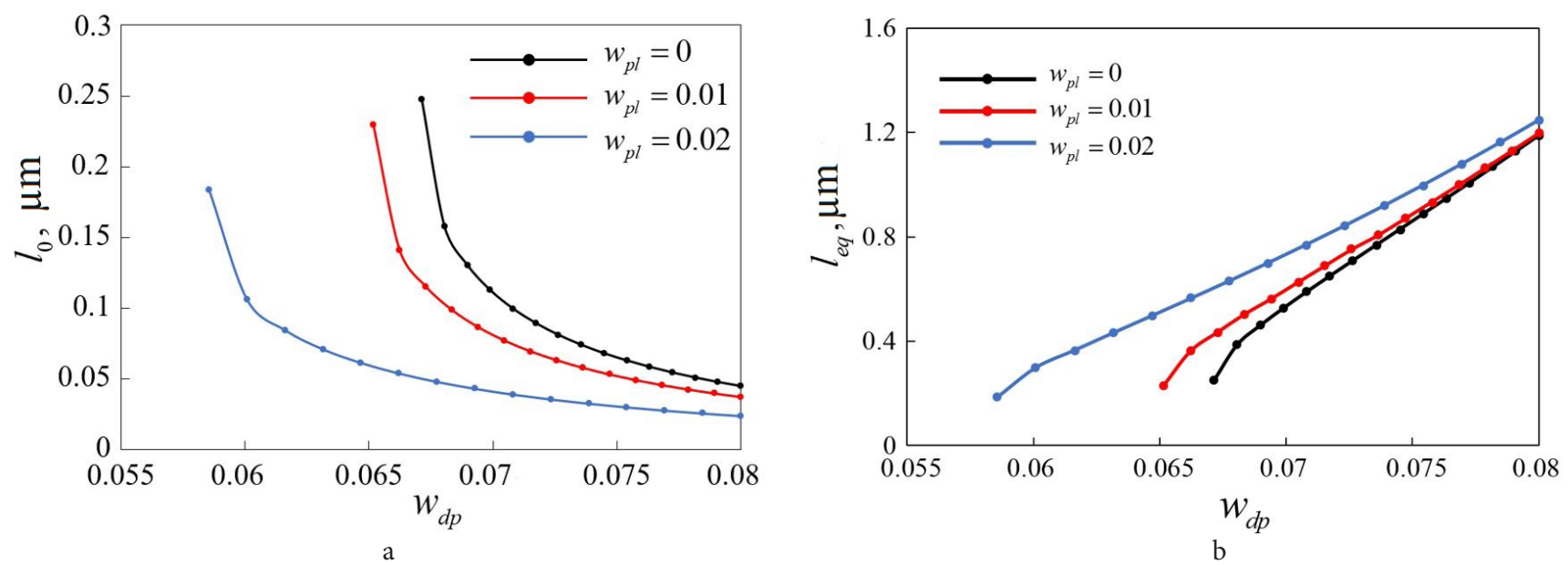

Pис. 4. (Color online) Зависимости длины зародышевой трещины $l_{0}$ (a) и длины стабильной трещины $l_{e q}$ (b) от мощности дисклинационного диполя при разных значениях мощности планарного мезодефекта $w_{p l}(2 a=0.6$ мкм).

Fig. 4. (Color online) Dependences of the length of the nuclear crack $l_{0}$ (a) and the length of the stable crack $l_{\text {eq }}$ (b) on the strength of the disclination dipole at different values of the power of the planar mesodefect $w_{p l}(2 a=0.6 \mu \mathrm{m})$.

Из рисунка видно, что с ростом мощности диполя дисклинаций и ростом мощности планарного мезодефекта длина зародышевой трещины уменьшается, а длина стабильной трещины становится больше. Влияние планарного мезодефекта уменьшается с ростом мощности дисклинационного диполя.

\section{4. Заключение}

Из проведённого анализа следует, что мезодефекты сдвигового типа могут существенно облегчать зарождение микротрещин в окрестности стыковых дисклинаций. Наличие планарного мезодефекта приводит к уменьшению длины зародышевой трещины и, следовательно, должно приводить к снижению энергии её появления. Кроме того, планарный мезодефект может заметно влиять на ориентацию и длину стабильных микротрещин. Следует отметить, что в процессе пластического деформирования материалов на характеристики микротрещины и ее стабильность влияют не только внутренние, но и внешние напряжения. Тем не менее проведенный в работе анализ при отсутствии внешнего напряжения может быть полезным в дальнейшем в плане сопоставления характеристик микротрещин, наблюдающихся вблизи стыков зерен и вершин фасеток на электронно-микроскопических изображениях после разгрузки образцов, претерпевших большие пластические деформации. При обсуждении применимости данной модели к реальным условиям пластического деформирования поликристаллических тел следует иметь ввиду, что она справедлива в том случае, когда затруднены процессы релаксации упругих полей напряжений от мезодефекта, связанные с аккомодационной пластической деформацией в его окрестности.

Благодарности / Aknowledgements. Исследование въполнено при финансовой поддержке Российского фонда фундаментальных исследований в рамках научного проекта №20-08-00867. I This work was supported by the Russian Foundation for Basic Research (project no. 20-08-00867).

\section{Литература/References}

1. V.V. Rybin. Large plastic deformations and fraction of metals. Moscow, Metallurgy (1986) 224 p. (in Russian) [В.В. Рыбин. Большие пластические деформации и разрушение металлов. Москва, Металлургия (1986) 224 с.]

2. V. V. Rybin, V. N. Perevezentsev, Y. V. Svirina. Phys. Metals Metallogr. 118 (12), 1171 (2017). Crossref

3. V. V. Rybin, I. M. Zhukovsky. Phys. Solid State. 20 (6), 1829 (1978). (in Russian) [В.В. Рыбин, И.. Жуковский. ФTT. 20 (6), 1829 (1978).]

4. M.S. Wu, K. Zhou, A.A. Nazarov. Phys. Rev. B. 76 (13), 134105 (2007). Crossref

5. G.F. Sarafanov, V.N. Perevezentsev. Def. and Fract. of Mat. 2, 2 (2016). (in Russian) [Г.Ф. Сарафанов, B.Н. Перевезенцев. Деформация и разрушение материалов. 2, 2 (2016).]

6. M.S. Wu. Int. J. Plast. 100, 142 (2018). Crossref

7. T. Wang, J. Luo, Z. Xiao, J. Chen. Eur. J. Mech. A. 28 (4), 688 (2009). Crossref

8. V. Rybin, V. Perevezentsev, S. Kirikov. Phys. Metals Metallogr. 119 (5), 421 (2018). Crossref

9. V.L. Indenbom. Phys. Solid State. 3, 2071 (1961). (in Russian) [В. Л. Инденбом. ФТТ. 3, 2071 (1961).]

10. G.F. Sarafanov, V.N. Perevezentsev, V.V. Rybin. Fundamentals of the kinetic theory of the formation of misoriented structures during plastic deformation of metals. N. Novgorod, Litera (2011) 359 p. (in Russian) [Г.Ф. Сарафанов, В.Н. Перевезенцев, В. В. Рыбин. Основы кинетической теории формирования разориентированных структур при пластической деформации металлов. Н. Новгород, Литера (2011) 359 с.]

11. V.A. Likhachev, R. Ю. Khayrov. Introduction to the theory of disclinations. Leningrad, Leningrad University (1975) 183 p. (in Russian) [В. А. Лихачев, Р. Ю. Хайров. Введение в теорию дисклинаций. Ленинград, Ленинградский университет (1975) 183 с.]

12. V.V. Rybin, A. N. Vergazov, Yu.F. Titovets. Surface. 6, 134 (1986). (in Russian) [В. В. Рыбин, А.Н. Вергазов, Ю. Ф. Титовец. Поверхность. 6, 134 (1986).] 